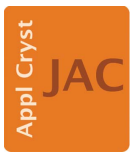

JOURNAL OF

APPLIED

CRYSTALLOGRAPHY

ISSN 1600-5767

Received 15 September 2016

Accepted 11 February 2017

Edited by A. J. Allen, National Institute of Standards and Technology, Gaithersburg, USA

Keywords: superparamagnetic nanoparticles; iron oxide; reference materials; SAXS; smallangle X-ray scattering; XANES; X-ray absorption near-edge structure; $X$-ray absorption fine structure.

Supporting information: this article has supporting information at journals.iucr.org/j

\section{SAXS analysis of single- and multi-core iron oxide magnetic nanoparticles}

\author{
Wojciech Szczerba, ${ }^{\mathrm{a}, \mathrm{b}}$ Rocio Costo, ${ }^{\mathrm{c}}$ Sabino Veintemillas-Verdaguer, \\ Maria del Puerto Morales ${ }^{c}$ and Andreas F. Thünemann ${ }^{\mathrm{a} *}$
}

${ }^{\mathbf{a}}$ Federal Institute for Materials Research and Testing (BAM), Unter den Eichen 87, 12205 Berlin, Germany, ${ }^{\mathbf{b}}$ Academic
Centre for Materials and Nanotechnology, AGH University of Science and Technology, Aleja Mickiewicza 30, 30-059
Kraków, Poland, and ' Instituto de Ciencia de Materiales de Madrid, ICMM/CSIC, Sor Juana Ines de la Cruz 3, 28049
Madrid, Spain. *Correspondence e-mail: andreas.thuenemann@bam.de

This article reports on the characterization of four superparamagnetic iron oxide nanoparticles stabilized with dimercaptosuccinic acid, which are suitable candidates for reference materials for magnetic properties. Particles $p_{1}$ and $p_{2}$ are single-core particles, while $\mathrm{p}_{3}$ and $\mathrm{p}_{4}$ are multi-core particles. Small-angle $\mathrm{X}$-ray scattering analysis reveals a lognormal type of size distribution for the iron oxide cores of the particles. Their mean radii are $6.9 \mathrm{~nm}\left(\mathrm{p}_{1}\right), 10.6 \mathrm{~nm}\left(\mathrm{p}_{2}\right)$, $5.5 \mathrm{~nm}\left(\mathrm{p}_{3}\right)$ and $4.1 \mathrm{~nm}\left(\mathrm{p}_{4}\right)$, with narrow relative distribution widths of 0.08 , $0.13,0.08$ and 0.12 . The cores are arranged as a clustered network in the form of dense mass fractals with a fractal dimension of 2.9 in the multi-core particles $p_{3}$ and $\mathrm{p}_{4}$, but the cores are well separated from each other by a protecting organic shell. The radii of gyration of the mass fractals are 48 and $44 \mathrm{~nm}$, and each network contains 117 and 186 primary particles, respectively. The radius distributions of the primary particle were confirmed with transmission electron microscopy. All particles contain purely maghemite, as shown by X-ray absorption fine structure spectroscopy.

\section{Introduction}

The synthesis, protection, functionalization and application of magnetic nanoparticles (MNPs) is a mature topic in nanochemistry (Lu et al., 2007). Currently, the probably most attractive applications of MNPs lie in the biomedical field (Karimi et al., 2013; Bohara et al., 2016).

Vital for their practical realization is to standardize, improve and redefine the analytical methods pertaining to MNPs, as is currently underway in the European research project with the acronym NanoMag (Bogren et al., 2105). To date there exists neither an MNP reference material nor a standard for defining properties of magnetic nanoparticles or characterization methods thereof. Although there are vendors on the market offering magnetic nanoparticle systems and labelling the magnetic properties with some numbers, these assertions cannot be verified since neither references nor standardized procedures exist to do so. Thus, a comparison of the product properties is virtually impossible for the customer. The situation for the characterization methods of magnetic properties of MNPs is no better. Results are strongly dependent on the experimental setup used, sample preparation and operation procedure, as well as the data reduction and analysis approach. The round robin studies performed so far are scarce and the results are deemed unpublishable. The situation would improve tremendously if there were a reference material addressing in a reproducible way at least one magnetic property, e.g. saturation magnetization, superparamagnetic 
blocking temperature or effective anisotropy constant. It is still under debate which of the many magnetic properties characterizes an MNP system most accurately for a specific application, but almost every one of them is related to the magnetic core size. One could relate the magnetic properties to energies instead of projecting them onto spheres of certain radii; this approach has not found its way into the MNP community and literature yet. Hence, the size of the magnetic cores and the distribution of core sizes remain among the most important characteristics of MNPs, which are used as an input for the determination and modelling of magnetic nanoparticles.

One can obtain the magnetic core sizes and their distribution from the analysis of magnetic data, for example, by analysing the high-field section of magnetization curves employing the Langevin function and assumed size distributions and shapes (Yoon, 2015). This information should be verified by complementary methods. Here, small-angle X-ray scattering (SAXS) is a natural solution for this problem. SAXS is capable of delivering representative and highly accurate data on the sizes of magnetic cores and on the distributions thereof. It provides incomparably more representative statistics on particle sizes and distributions than imaging techniques like transmission electron microscopy (TEM) or scanning electron microscopy, although those techniques are clearly better at determining the shapes of the cores. SAXS has a good reproducibility of the results, as the averaging depends on a physical effect rather than the judgement of the experimenter analysing an image and arbitrarily assigned particle size definitions.

Here, we report on a selection of four of the MNP systems under investigation, foreseen as reference materials for different magnetic properties that are designed to be biocompatible too. All four are stabilized with dimercaptosuccinic acid (DMSA) (Odio et al., 2014). The ligand exchange process of oleic acid by DMSA results in MNP systems that are single-core particles (denoted $\mathrm{p}_{1}$ and $\mathrm{p}_{2}$ ) or display a multicore structure $\left(\mathrm{p}_{3}\right.$ and $\left.\mathrm{p}_{4}\right)$. An MNP reference material must have reproducible magnetic properties of interest. Since the magnetism of nanoparticles depends strongly on core sizes, shapes and crystalline structure, as well as aggregation behaviour, the reproducibility and reliable characterization of these parameters must be addressed first.

\section{Experimental}

\subsection{Synthesis of magnetic nanoparticles}

The procedure to obtain uniform magnetic nanoparticles with controlled core diameter by thermal decomposition and its transference to water takes place in three steps: (i) synthesis of the iron oleate precursor, (ii) synthesis of iron oxide magnetic nanoparticles and (iii) transference to water by ligand exchange. The synthesis was carried out following the procedure previously described by Salas et al. (2012) In brief, first, iron oleate was prepared by dissolving $\mathrm{FeCl}_{3} \cdot 6 \mathrm{H}_{2} \mathrm{O}$ salt in water and adding it to a solution made of sodium oleate, ethanol and hexane. This mixture was heated at around $343 \mathrm{~K}$ for $4 \mathrm{~h}$, washed with distilled water and ethanol in a funnel, and left to dry for two days. The iron content was $6 \%$. Then, $4.5 \mathrm{~g}$ of the iron oleate was mixed with oleic acid in $50 \mathrm{ml}$ of octadecene. The amount of oleic acid was varied between 0.6 and $1.2 \mathrm{~g}$, leading to oleic acid/Fe molar ratios of around 4-3 to obtain particle sizes between 20 and $7 \mathrm{~nm}$. The mixture was heated to $593 \mathrm{~K}$ at $3 \mathrm{~K} \mathrm{~min}^{-1}$. Finally, the particles were transferred to water by ligand exchange reaction with DMSA in a mixture of toluene and dimethylsulphoxide (Roca et al., 2009). Applying this procedure, two single-core MNP systems, denoted as $\mathrm{p}_{1}$ and $\mathrm{p}_{2}$, and two multi-core MNP systems, $\mathrm{p}_{3}$ and $\mathrm{p}_{4}$, were produced. The production of single-core or multicore particles depends on the efficiency of the ligand exchange process, which depends on the amount of oleic acid on the particle surface and the DMSA added (90 mg DMSA $/ 50 \mathrm{mg}$ $\mathrm{Fe})$. In this work, the ligand exchange process was more efficient for the largest cores $(>12 \mathrm{~nm}$ in diameter) owing to the reduction in specific surface area (lower amount of oleic acid on the nanoparticle surface). Larger amounts of DMSA (200 mg DMSA/50 mg Fe) are expected to lead to single-core particles for samples with smaller sizes $(<10 \mathrm{~nm}$ in diameter).

\subsection{SLS - static light scattering}

The SLS experiments were performed using a multi-angle detector setup equipped with an $\mathrm{He}-\mathrm{Ne}$ laser from ALV, Langen, Germany. The samples were diluted by factors of 100 , 200, 500, 1000 and 2000 to obtain a dilution series. The SLS data were converted to $I(q)$ sets, by using the expression $q=$ $(4 \pi n / \lambda) \sin \theta$ with $\lambda=632.8 \mathrm{~nm}(\theta$ being half the scattering angle and $n$ is the refractive index).

2.2.1. SAXS measurements. SAXS measurements were performed in a flow-through capillary with a Kratky-type instrument (SAXSess from Anton Paar, Austria) at $294 \pm 1$ K. The SAXSess has a low sample-to-detector distance of $0.309 \mathrm{~m}$, which is appropriate for investigation of dispersions with low scattering intensities. The samples were measured as delivered after vortexing for $3 \mathrm{~min}$. The measured intensity was converted to an absolute scale according to Orthaber $e t a l$. (2000). The scattering vector magnitude $q$ depends on the wavelength $\lambda$ of the radiation $(\lambda=0.154 \mathrm{~nm})$ as $q=(4 \pi n /$ $\lambda) \sin \theta$. Deconvolution (slit length desmearing) of the SAXS curves was performed with the SAXS-Quant software (Anton Paar). Samples analysed with SAXS were used as prepared. Curve fitting was conducted with the software SASfit (Bressler et al., 2015).

\subsection{XANES - X-ray absorption near-edge structure}

The X-ray absorption fine structure (XAFS) experiments were carried out at the BAMline (Görner et al., 2001; Riesemeier et al., 2005) with the BESSY II synchrotron light source in Berlin, Germany. The XAFS spectra at the $K$ edge of iron $(7112 \mathrm{eV})$ were recorded in transmission mode using two ionization chambers (Oxford Danfysik IC Plus 50). The incident beam intensity was monitored using an ionization chamber filled with air at ambient pressure, giving a 
transmission rate of 0.92 at $7100 \mathrm{eV}$. The absorption signal was measured using a second ionization chamber of the same type, filled with argon gas at ambient pressure, with a transmission rate of approximately 0.25 in the energy range of interest. The energy was scanned using an $\mathrm{Si}(111)$ double-crystal monochromator with a relative energy resolution of $5 \times 10^{-5}$. The XAFS scans in the near-edge region (XANES) were carried out in the range from $7032 \mathrm{eV}(80 \mathrm{eV}$ below the edge) to $7182 \mathrm{eV}(70 \mathrm{eV}$ above the edge) with a step of $1 \mathrm{eV}$. The edge jump was measured with a finer step of $0.5 \mathrm{eV}$. The extended absorption fine structure region (EXAFS) was scanned up to $k_{\max }=12 \AA^{-1}$ above the absorption edge with a large step in the momentum space to make the normalization of the spectra more convenient. The scans were recorded with an acquisition time of $4 \mathrm{~s}$ per point. The spectra were calibrated to the absorption edge energy of metallic iron, $7112 \mathrm{eV}$. The energy step in the XANES region was smaller than the natural broadening caused by the finite mean lifetime of the excitation states.

\subsection{TEM imaging}

Particle sizes and shapes were studied by TEM using a JEOL JEM 1010 microscope operated at $100 \mathrm{keV}$. TEM samples were prepared by placing one drop of a dilute particle suspension on an amorphous carbon-coated copper grid and evaporating the solvent at room temperature. The mean particle size and distribution were evaluated by measuring the largest internal dimension of at least 100 particles. Afterwards, data were fitted to a lognormal distribution, obtaining the mean size $(\bar{x})$ and the standard deviation $(\sigma)$.

\section{Results and discussion}

The spherical magnetic nanoparticle systems under investigation can be divided into two groups according to their coarse morphology. These are single-core and multi-core particles (Lartigue et al., 2012). The single-core particles consist of isolated cores with functionalized coatings, whereas the multi-core particles are composed of cores that strongly interact with each other within agglomerates. When interpreting their magnetic properties, we regard these agglomerates of interacting cores as single magnetic nanoparticles. The particles denoted $\mathrm{p}_{1}$ and $\mathrm{p}_{2}$ are single-core particles, whereas $\mathrm{p}_{3}$ and $\mathrm{p}_{4}$ are multi-core ones judging by the routes of syntheses chosen by manufacturers and the available TEM data.

Here, SAXS was employed to determine the sizes of the particle cores and the distribution thereof. The intensity of the SAXS signal is proportional to the square of the density difference between the solvent and the object of interest. The density of $\mathrm{Fe}_{2} \mathrm{O}_{3}$ is approximately $4.9 \mathrm{~g} \mathrm{~cm}^{-3}$, while the density of DMSA is about $1.6 \mathrm{~g} \mathrm{~cm}^{-3}$. The solvent is water with $1.0 \mathrm{~g} \mathrm{~cm}^{-3}$. The contrast is the ratio of these squared differences $\left(3.9^{2} / 0.6^{2}\right)$. Hence, the iron oxide cores scatter X-rays about 50 times stronger than the organic shell made of dimercaptosuccinic acid. Therefore, we 'see' only the iron oxide with SAXS. The scattering intensity, $I(q)$, of particles in the monodisperse approximation is given as the product of the particle form factor, $P(q)$, and the structure factor, $S(q)$, as

$$
I(q)=P(q) S(q) .
$$

In this work we model the single-core particles with $S(q)=1$, which means that no interaction is present between the particles. In contrast, the structure factor of the multi-core particle is $S(q) \geq 1$.

\section{Single-core particles}

The SAXS curve of $\mathrm{p}_{1}$ displays characteristically sharp minima at $q=0.64 \mathrm{~nm}^{-1}$ and $q=1.11 \mathrm{~nm}^{-1}$, which is a typical indication of particles with a very narrow size distribution (Bonini et al., 2007). The scattering fringes of the SAXS curve of $\mathrm{p}_{2}$ are less pronounced than those for $\mathrm{p}_{1}$. Hence, the relative size distribution of the particle cores is broader. The first minimum at $q=0.42 \mathrm{~nm}^{-1}$ sets in at a lower $q$ value than for $\mathrm{p}_{1}$, indicating that the particle cores of $p_{2}$ are larger than those of $p_{1}$ (see circles and squares, respectively, in Fig. 1). We found that the scattering curves are reproduced well using the analytical form factor of a sphere, $I_{\mathrm{s}}(q, R, \Delta \eta)$, with a lognormal number-weighted size distribution of the radii [the use of this distribution function is justified in the supporting information; for further literature relating to this material, see O'Grady \& Bradbury (1983) and Hamilton (1965)], $f\left(R, N, \sigma, R_{\text {median }}\right)$, as

$$
I(q)=\int_{0}^{\infty} f\left(R, N, \sigma, R_{\text {median }}\right) I_{\mathrm{s}}(q, R, \Delta \eta) \mathrm{d} R .
$$

The lognormal size distribution is defined as

$$
f\left(N, \sigma, R, R_{\text {median }}\right)=\frac{N}{(2 \pi)^{1 / 2} \sigma R} \exp \left[-\frac{\ln \left(R / R_{\text {median }}\right)^{2}}{2 \sigma^{2}}\right]
$$

and the scattering of a single sphere is given by

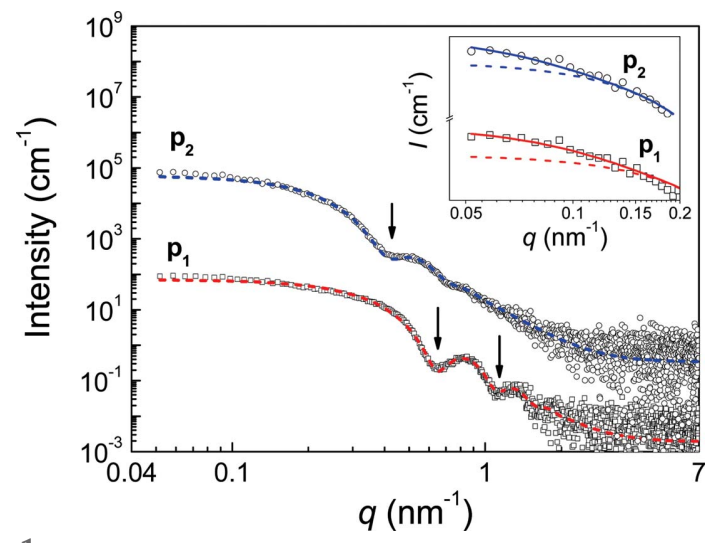

Figure 1

SAXS curve of single-core particles $\mathrm{p}_{1}$ and $\mathrm{p}_{2}$ (squares and circles, respectively). Data of $\mathrm{p}_{2}$ are multiplied by $10^{3}$ for better visibility. Curve fits utilizing a lognormal size distribution of the radii are given (red and blue dashed lines, respectively). Minima at $q=0.64 \mathrm{~nm}^{-1}$ and $q=$ $1.11 \mathrm{~nm}^{-1}$ for $\mathrm{p}_{1}$ and at $q=0.42 \mathrm{~nm}^{-1}$ for $\mathrm{p}_{2}$ are indicated by arrows. Inset. Enlargement of the low- $q$ range with addition of the scattering contribution of the small fraction of aggregates (solid curves). 


$$
I_{\mathrm{s}}(q, R, \Delta \eta)=\left\{\frac{4}{3} \pi R^{3} \Delta \eta\left[3 \frac{\sin (q R)-q R \cos (q R)}{(q R)^{3}}\right]\right\}^{2} .
$$

$R$ is the sphere radius and $\Delta \eta$ the scattering length density difference between the particle and the matrix. For iron oxides in aqueous solution $\Delta \eta$ is $2.8974 \times 10^{-11} \mathrm{~cm}^{-2}$ when presuming an $\mathrm{Fe}_{2} \mathrm{O}_{3}$ stoichiometry, a density of $4.9 \mathrm{~g} \mathrm{~cm}^{-3}$ and an X-ray energy of $8.6 \mathrm{keV} . \sigma$ is the width parameter of the size distribution and $R_{\text {median }}$ is the median radius. $N$ is the particle number density. Using these parameters, the mean radius was calculated as $R_{\text {mean }}=R_{\text {median }} \exp \left(\sigma^{2}\right) / 2$ and the relative standard deviation of the width of the size distributions as $\sigma_{\text {width }} / R_{\text {mean }}=\left[\exp \left(2 \sigma^{2}\right)-\exp \left(\sigma^{2}\right)\right]^{1 / 2}$.

Equation (1) was employed for interpretation of the scattering data of $\mathrm{p}_{1}$ and $\mathrm{p}_{2}$, resulting in the fitted curves shown in Fig. 1 (red and blue dashed lines, respectively). The distributions of the particles' number density in particles per $\mathrm{cm}^{3}$ as a probability density function (PDF) and a cumulative density function (CDF) are shown in Fig. 2(a) (solid and dashed curves, respectively). We calculated the volume-weighted PDF and $\mathrm{CDF}$ in terms of iron concentration for comparison (Fig. $2 b$, solid and dashed curves). The mean radii are $R_{\text {mean }}=$ $6.9 \pm 0.1 \mathrm{~nm}\left(\mathrm{p}_{1}\right)$ and $10.6 \pm 0.1 \mathrm{~nm}\left(\mathrm{p}_{2}\right)$, and the relative distribution width are $0.08 \pm 0.01$ and $0.13 \pm 0.02$. Additionally, the total particle number densities are $N=(4.2 \pm 0.2) \times$ $10^{14} \mathrm{~cm}^{-3}$ and $N=(2.4 \pm 0.2) \times 10^{14} \mathrm{~cm}^{-3}$ (see Fig. $2 a$ ). Calculation of the volume fraction for $\mathrm{p}_{1}$ results in $\varphi=(5.6 \pm$ $0.2) \times 10^{-4}$, which corresponds to a total iron oxide concentration of $c=2.7 \pm 0.1 \mathrm{mg} \mathrm{ml}^{-1}$ and a total iron content of $1.9 \pm 0.1 \mathrm{mg} \mathrm{ml}^{-1}$. Similarly, the volume fraction of $\mathrm{p}_{2}$ is $\varphi=$ $(1.1 \pm 0.1) \times 10^{-3}$, corresponding to a total iron oxide concentration of $c=5.6 \pm 0.2 \mathrm{mg} \mathrm{ml}^{-1}$ and a total iron content of $4.0 \pm 0.2 \mathrm{mg} \mathrm{ml}^{-1}$.

The low- $q$ range of the SAXS curves exhibits a plateau toward zero, indicating that there are hardly any particles or agglomerates present in the sample that are significantly larger than the primary particle cores. However, the model fit with the primary particles for $\mathrm{p}_{1}$ very slightly underestimates the intensity of the experimental curve at $q<0.1 \mathrm{~nm}^{-1}$. The addition of a small population of larger spherical particles corrects this mismatch (see inset of Fig. 1). The spheres have a mean radius of about $21 \mathrm{~nm}$ and a particle number density of about $2 \times 10^{11} \mathrm{~cm}^{-3}$. We interpret the larger structures as aggregates consisting of the smaller particles. The volume ratio indicates that there are around 30 primary particles contained in one aggregate on average. Furthermore, from the fitted intensity coefficients one can state that there are 2000 times more primary particles than aggregates. Most probably the cores are in direct contact within the aggregate and therefore SAXS cannot resolve the inner structure of these.

As in the case of $p_{1}$, there is a small mismatch of the intensities of $\mathrm{p}_{2}$ between the model and the experiment at $q<$ $0.1 \mathrm{~nm}^{-1}$. The addition of a small population of larger spheres corrects the underestimation in this case, too. Like for $p_{1}$ we interpret this as the presence of a few aggregates consisting of the primary particle cores. The aggregates have a mean radius of about $28 \mathrm{~nm}$. The particle number density is about $4 \times 10^{11} \mathrm{~cm}^{-3}$. This means that one aggregate consists of approximately 20 primary particles. The ratio of the fitted intensity factors for the two populations suggests that there is one aggregate per 540 primary particles.

\section{Multi-core particles}

Visual inspection of the scattering curves of $\mathrm{p}_{3}$ and $\mathrm{p}_{4}$ reveals similar curve shapes as for $\mathrm{p}_{1}$ and $\mathrm{p}_{2}$ for $q$ values larger than about $0.5 \mathrm{~nm}^{-1}$, as shown in Fig. 3. However, the low- $q$ part of the SAXS curve between 0.05 and $0.10 \mathrm{~nm}^{-1}$ has a steep rise toward zero. This is a clear sign of larger structure motifs present in the sample in significant numbers. To make an estimate of these large structures, the SAXS curves were combined with static light scattering data to obtain additional scattering data in an interval of $0.59 \times 10^{-2} \leq q \leq 2.60 \times$ $10^{-2} \mathrm{~nm}^{-1}$. The SLS and SAXS curves have different intensities. The SAXS curve is plotted in absolute units, whereas the SLS curve is plotted in arbitrary units that are normalized to

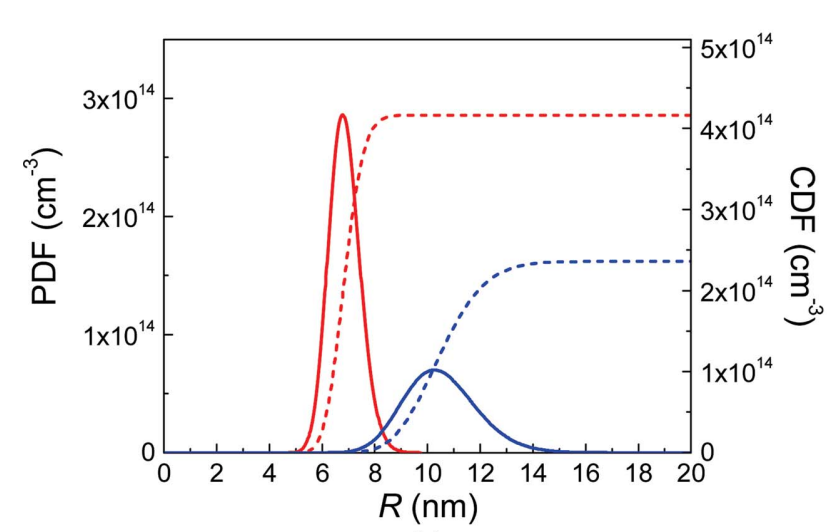

(a)

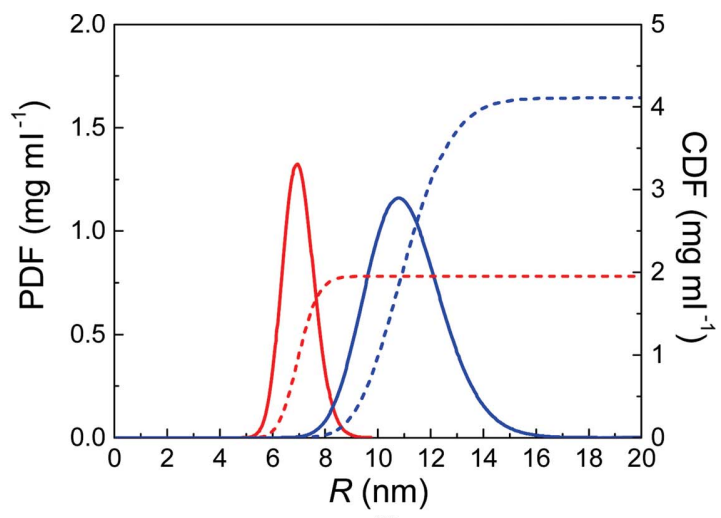

(b)

Figure 2

Size distributions of $\mathrm{p}_{1}$ and $\mathrm{p}_{2}$ (red and blue lines) in number-weighted and volume-weighted form $[(a)$ and $(b)$, respectively]. Displayed are the partial differential functions (solid lines) and cumulative distribution functions (dashed lines). The number-weighted distributions are given in units of particles per $\mathrm{cm}^{3}$. The volume-weighted size distributions in $(b)$ are given in units of iron content in $\mathrm{mg}$ per ml, calculated from the particles' volume fraction, a particle density of $4.9 \mathrm{~g} \mathrm{~cm}^{-3}$ and an $\mathrm{Fe}_{2} \mathrm{O}_{3}$ stoichiometry. 


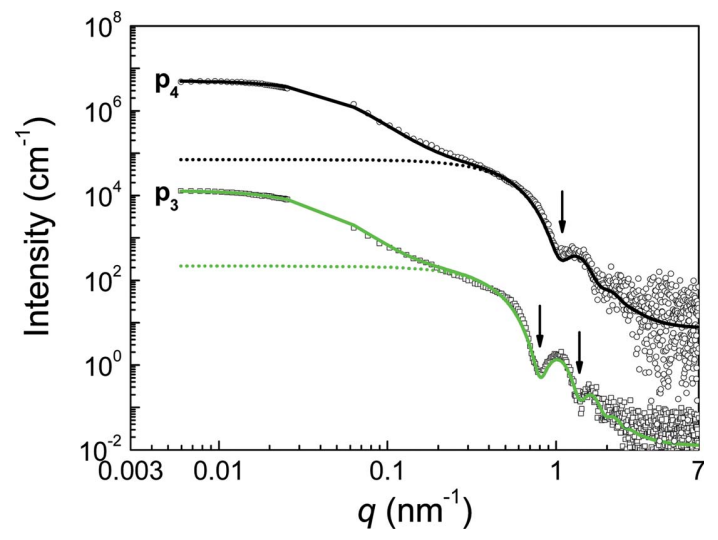

Figure 3

The combined SAXS and SLS curves of multi-core particles $\mathrm{p}_{3}$ and $\mathrm{p}_{4}$ (squares and circles, respectively). Data of $\mathrm{p}_{4}$ are multiplied by $10^{3}$ for better visibility. Curve fits according to equation (1) are given (solid lines). The mass fractal structure factor in equation (4) is used for consideration of the multi-core structure. Minima at $q=0.82 \mathrm{~nm}^{-1}$ and $q=$ $1.38 \mathrm{~nm}^{-1}$ for $\mathrm{p}_{3}$ and at $q=1.03 \mathrm{~nm}^{-1}$ for $\mathrm{p}_{4}$ are indicated by arrows. The scattering profiles of the particles alone without structure factor are given as dotted lines.

the value of the first point. Additionally, the ranges of the curves do not overlap; there is a gap that makes merging of the data not straightforward. The solution to the problem is to use the capability of SASfit to fit multiple data sets simultaneously, which is usually employed for small-angle neutron scattering (Bressler et al., 2015). The fits are carried out with two independent parameters for the scattering intensities of the two sets; the other parameters are common for both sets of data. The ratio of the intensity parameter is used to scale the SLS curve intensity, so that it matches the intensity of SAXS. Both sets are merged into one, which is fitted again as a single data set to check for the correctness of the procedure.

The combined data of static light scattering and SAXS in Fig. 3 show a plateau towards zero. Application of Guinier's law (Guinier \& Fournet, 1955), $I(q)=I_{0} \exp \left(-\frac{1}{3} R_{\mathrm{g}}^{2} q^{2}\right)$, to the light scattering data provides radii of gyration of $R_{\mathrm{g}}=48 \pm$ $1 \mathrm{~nm}$ for $\mathrm{p}_{3}$ and $R_{\mathrm{g}}=44 \pm 1 \mathrm{~nm}$ for $\mathrm{p}_{4}$.

Tentatively, we interpret the larger objects as fractal agglomerates of primary particles. Accordingly, the interparticle structure factor (Ferretti et al., 1998; Teixeira, 1988)

$$
\begin{aligned}
& S(q)=1+\frac{D}{r_{0}^{D}} \int_{0}^{\infty} r^{D-3} \exp \left[-\left(\frac{r}{\xi}\right)\right] \frac{\sin (q r)}{q r} r^{2} \mathrm{~d} r, \\
& S(q)=1+\left(\frac{\xi}{r_{0}}\right)^{D} \Gamma(D+1) \frac{\sin \left[(D-1) \tan ^{-1}(q \xi)\right]}{(D-1) q \xi\left[1+(q \xi)^{2}\right]^{(D-1) / 2}}
\end{aligned}
$$

was found appropriate for interpretation of the scattering curves of $p_{3}$ and $p_{4}$ by multiplying the form factor of the primary particle with the fractal structure factor according to equation (1). The results are displayed in Fig. 3. The parameter $D$ in equations (5) and (6) is the fractal dimension, and $r_{0}$ is the radius of the individual particles making up the fractal aggregate. Here, we interpret $r_{0}$ as the radius of the primary particle core radius plus a shell thickness, which is formed by dimercaptosuccinic acid molecules. $\xi$ is the characteristic size of the fractal or more precisely the cutoff length for the fractal correlation. It represents the distance above which the mass distribution in the aggregate is no longer described by the fractal law. Here the exponential cutoff criterion $\exp [-(r / \xi)]$ is used.

Similarly as in the case of the single-core $\mathrm{p}_{1}$, the SAXS curve of multi-core $\mathrm{p}_{3}$ shows two sharp minima, at $q=$ $0.82 \mathrm{~nm}^{-1}$ and $q=1.38 \mathrm{~nm}^{-1}$, indicating spherical particles with a very narrow size distribution. The SAXS curve of $\mathrm{p}_{4}$ has a very similar shape to that of $\mathrm{p}_{3}$ at high $q$ values (see Fig. 3). The minimum is blurred, indicating a wider relative size distribution than for $\mathrm{p}_{3}$. The position of the minimum at $1.0 \mathrm{~nm}^{-1}$ means that the mean core radius is the smallest of the whole series of samples. In the high- $q$ range the scattering curve is well reproduced using the form factor of a sphere with lognormal size distribution of moderate width. The best fit results are mean core radii of $R=5.5 \pm 0.1 \mathrm{~nm}$ and

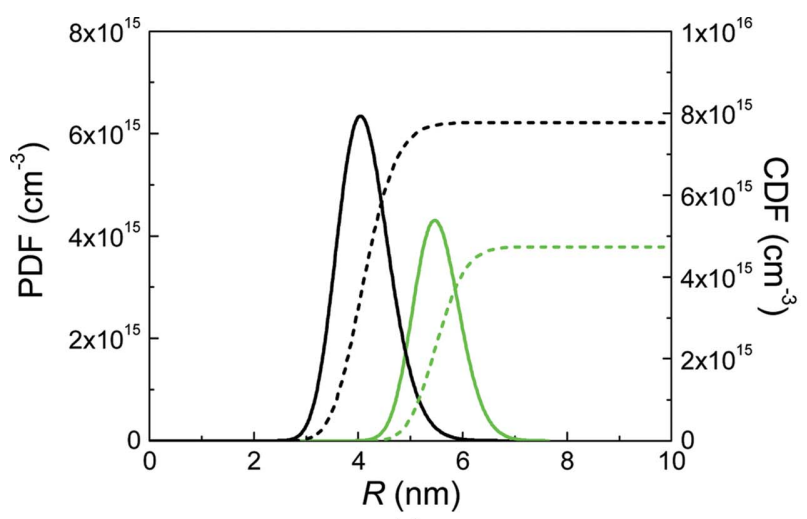

(a)

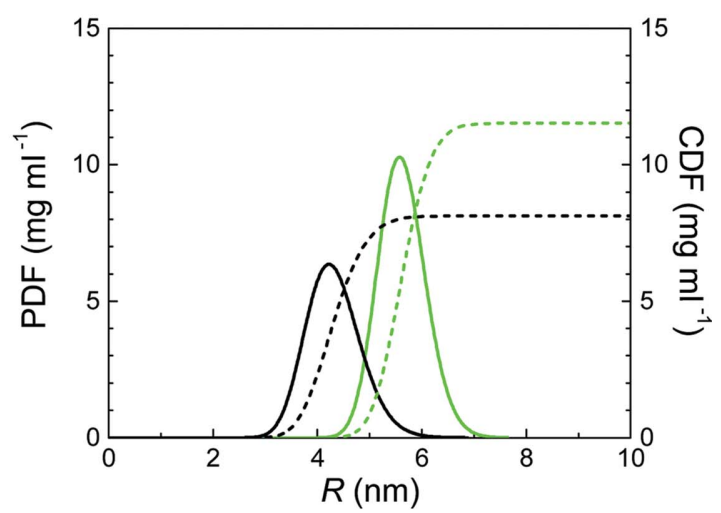

(b)

Figure 4

Size distributions of the cores of the primary particles of $\mathrm{p}_{3}$ and $\mathrm{p}_{4}$ (green and black lines) in number-weighted and volume-weighted form $[(a)$ and $(b)$, respectively]. Displayed are the partial differential functions (solid lines) and cumulative distribution functions (dashed lines). The number-weighted distributions are given in units of particles per $\mathrm{cm}^{3}$. The volume-weighted size distributions in $(b)$ are given in units of iron content in mg per ml, calculated on the basis of an $\mathrm{Fe}_{2} \mathrm{O}_{3}$ stoichiometry. 
Table 1

Summary of the particle characteristics derived from scattering methods.

$R_{\mathrm{g}, \mathrm{LS}}$ is the radius of gyration derived from static light scattering data, $R$ is the mean radius of the core of the primary particle, $\sigma / R$ is the relative width of the size distribution, $r_{0}$ is the mean radius of a primary particle of core and shell within a multi-core particle, $\xi$ is the cutoff length for the fractal correlation, $D$ is the mass fractal dimension of the multi-core particles, and $N_{\text {agg }}$ is the aggregation number of a multi-core particle. Values in parentheses are uncertainties on the least significant digit.

\begin{tabular}{|c|c|c|c|c|c|c|c|}
\hline \multirow[b]{2}{*}{ Nanoparticles } & \multirow[b]{2}{*}{$R_{\mathrm{g}, \mathrm{LS}}(\mathrm{nm})$} & \multicolumn{2}{|l|}{ Cores } & \multicolumn{4}{|c|}{ Mass fractal aggregates } \\
\hline & & $R(\mathrm{~nm})$ & $\sigma / R$ & $r_{0}(\mathrm{~nm})$ & $\xi(\mathrm{nm})$ & $D$ & $N_{\text {agg }}$ \\
\hline \multicolumn{8}{|c|}{ Single-core particles $\dagger$} \\
\hline $\mathrm{p}_{1}$ & - & 6.9 (1) & 0.08 (1) & - & - & - & - \\
\hline $\mathrm{p}_{2}$ & - & $10.5(1)$ & $0.13(2)$ & - & - & - & - \\
\hline \multicolumn{8}{|c|}{ Multi-core particles } \\
\hline $\mathrm{p}_{3}$ & 48 (1) & $5.5(1)$ & 0.08 (1) & $9.3(2)$ & $22(2)$ & $2.9(1)$ & 117 (16) \\
\hline $\mathrm{p}_{4}$ & 44 (1) & 4.1 (1) & $0.12(2)$ & $6.8(2)$ & $18(2)$ & $2.8(1)$ & $186(27)$ \\
\hline
\end{tabular}

$\dagger$ An amount of less than $1 \%$ of primary particles is present in aggregates.

$R=4.1 \pm 0.1 \mathrm{~nm}$ for $\mathrm{p}_{3}$ and $\mathrm{p}_{4}$, respectively. The relative widths of the size distributions are $0.08 \pm 0.01$ and $0.12 \pm 0.02$. The scattering data without structure factor contribution are shown separately in Fig. 3. The corresponding distributions of the particle number densities (PDF and CDF) are shown in Fig. 4(a) (solid and dashed curves, respectively). We calculated the volume-weighted PDF and CDF in terms of iron concentration for comparison (Fig. 4b). Additionally, the total particle number densities are $N=(4.7 \pm 0.5) \times 10^{15} \mathrm{~cm}^{-3}$ and $N=(7.7 \pm 0.2) \times 10^{15} \mathrm{~cm}^{-3}$ (see Fig. 4a). Calculation of the volume fraction for $\mathrm{p}_{3}$ results in $\varphi=(3.3 \pm 0.1) \times 10^{-3}$, which corresponds to a total iron oxide concentration of $c=16.3 \pm$ $0.2 \mathrm{mg} \mathrm{ml}^{-1}$ and a total iron content of $11.4 \pm 0.2 \mathrm{mg} \mathrm{ml}^{-1}$. Similarly, the volume fraction of $\mathrm{p}_{4}$ is $\varphi=(2.2 \pm 0.1) \times 10^{-3}$,

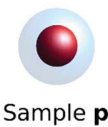

Sample $\mathbf{p}_{1}$

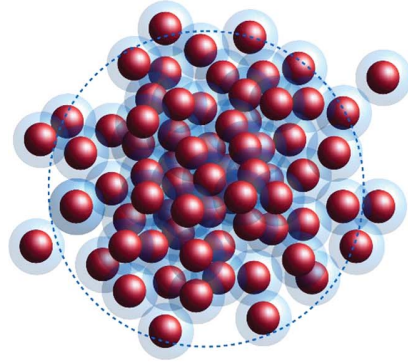

Sample $\mathbf{p}$
Sample $\mathbf{p}_{2}$
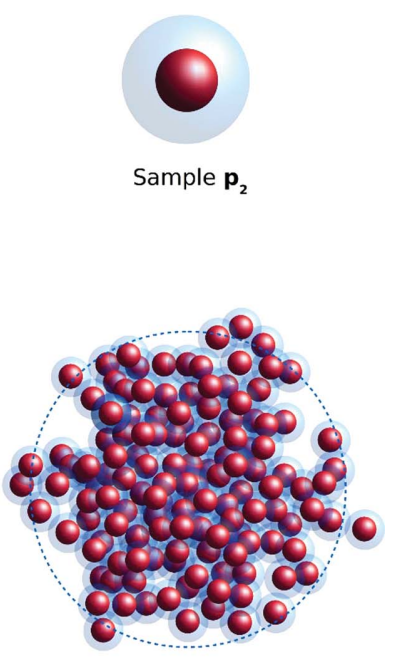

Sample $\mathbf{p}_{4}$
Figure 5

Schematic pictures of single-core $\left(\mathrm{p}_{1}, \mathrm{p}_{2}\right)$ and multi-core particles $\left(\mathrm{p}_{3}, \mathrm{p}_{4}\right)$. The primary particles consisting of iron oxide are displayed as solid spheres and their stabilizing shells are transparent blue. The blue dashed lines in the structures of $\mathrm{p}_{3}$ and $\mathrm{p}_{4}$ indicate the characteristic size of the fractals. corresponding to a total iron oxide concentration of $c=11.2 \pm$ $0.2 \mathrm{mg} \mathrm{ml}^{-1}$ and a total iron content of $7.9 \pm 0.1 \mathrm{mg} \mathrm{ml}^{-1}$.

The best fit was achieved with a fractal dimension of $D=$ $2.9 \pm 0.1$ for $\mathrm{p}_{3}$ and $2.8 \pm 0.1$ for $\mathrm{p}_{4}$. Therefore, the fractal dimensions of $\mathrm{p}_{3}$ and $\mathrm{p}_{4}$ are the same within the experimental accuracy. Such large values of $D$ close to 3 represent compact mass fractal structures. The finding of high fractal dimensions indicates that both multi-core particles are produced by a reaction-limited colloidal aggregation that displays $D$ values $>$ 2 (Lin et al., 1989). The values for $\xi$ are $22 \pm 2 \mathrm{~nm}$ for $\mathrm{p}_{3}$ and $18 \pm 2 \mathrm{~nm}$ for $\mathrm{p}_{4}$. The radii $r_{0}$ are $9.3 \pm 0.2 \mathrm{~nm}$ and $6.8 \pm$ $0.2 \mathrm{~nm}$. The difference of $r_{0}$ and $R$ can be interpreted as the thickness of the organic coating of the particles. This assumption results in estimates of $3.9 \pm 0.2 \mathrm{~nm}$ for the thickness of the organic coating of $p_{3}$ and $2.7 \pm 0.2 \mathrm{~nm}$ for $p_{4}$. These values indicate a thicker coating for $\mathrm{p}_{3}$ than for $\mathrm{p}_{4}$. The presence of this coating, which is obviously much thicker than a monolayer thickness of the stabilizer, suggests also that the primary particles are isolated from each other by the organic material of the coating.

We checked the consistency of our results. For this purpose we used the fact that the radius of gyration can also be determined from the fit parameters (Ferretti et al.,1998) of the fractal as

$$
R_{\mathrm{g}, \text { fractal }}=\xi\left[\frac{1}{2} D(D+1)\right]^{1 / 2}
$$

resulting in $R_{\mathrm{g}, \text { fractal }}=52 \pm 5 \mathrm{~nm}$ for $\mathrm{p}_{3}$ and $42 \pm 5 \mathrm{~nm}$ for $\mathrm{p}_{4}$. These values are in agreement with the $R_{\mathrm{g}}$ values from utilizing Guinier's law (see Table 1). The number of primary particles can be estimated as (Teixeira, 1988)

$$
N_{\text {agg }}=\left(R_{\mathrm{g}} / r_{0}\right)^{D},
$$

resulting in $117 \pm 16$ and $186 \pm 27$ primary particles per aggregate. The fit parameters are summarized in Table 1 for ease of comparison. A schematic picture of the particles' structures is shown in Fig. 5.

\section{Comparison with TEM}

TEM measurements were conducted in order to check our findings from SAXS. The TEM results are summarized in Fig. 6 . The particle radius distributions were derived from the pictures in the insets in the form of histograms. Fits of lognormal functions in PDF and CDF presentation are displayed as solid and dashed lines, respectively. It should be noted that it is hardly possible to distinguish aggregated from non-agglomerated particles by TEM unless it is performed in solution using Cryo-TEM. But such an investigation is difficult to conduct. In addition, it is time-consuming and therefore expensive work to count a large enough number of particles in order to obtain representative radius distributions. Here, the number of particles is of the order of 100 as can be seen from the cumulative sum of the particles (right axes). Nevertheless, fitting of the TEM data with lognormal distributions provides mean radii of $7.0 \mathrm{~nm}\left(\mathrm{p}_{1}\right), 9.3 \mathrm{~nm}\left(\mathrm{p}_{2}\right), 5.7 \mathrm{~nm}\left(\mathrm{p}_{3}\right)$ and $3.9 \mathrm{~nm}$ $\left(\mathrm{p}_{4}\right)$. The relative widths of the radius distributions are 0.12 
$\left(\mathrm{p}_{1}\right), 0.10\left(\mathrm{p}_{2}\right), 0.10\left(\mathrm{p}_{3}\right)$ and $0.17\left(\mathrm{p}_{4}\right)$. Comparison of these TEM results with the summarized values for the primary particles in Table 1 reveals an excellent agreement of TEM and SAXS values. We therefore conclude that the TEM measurements confirm our results from SAXS.
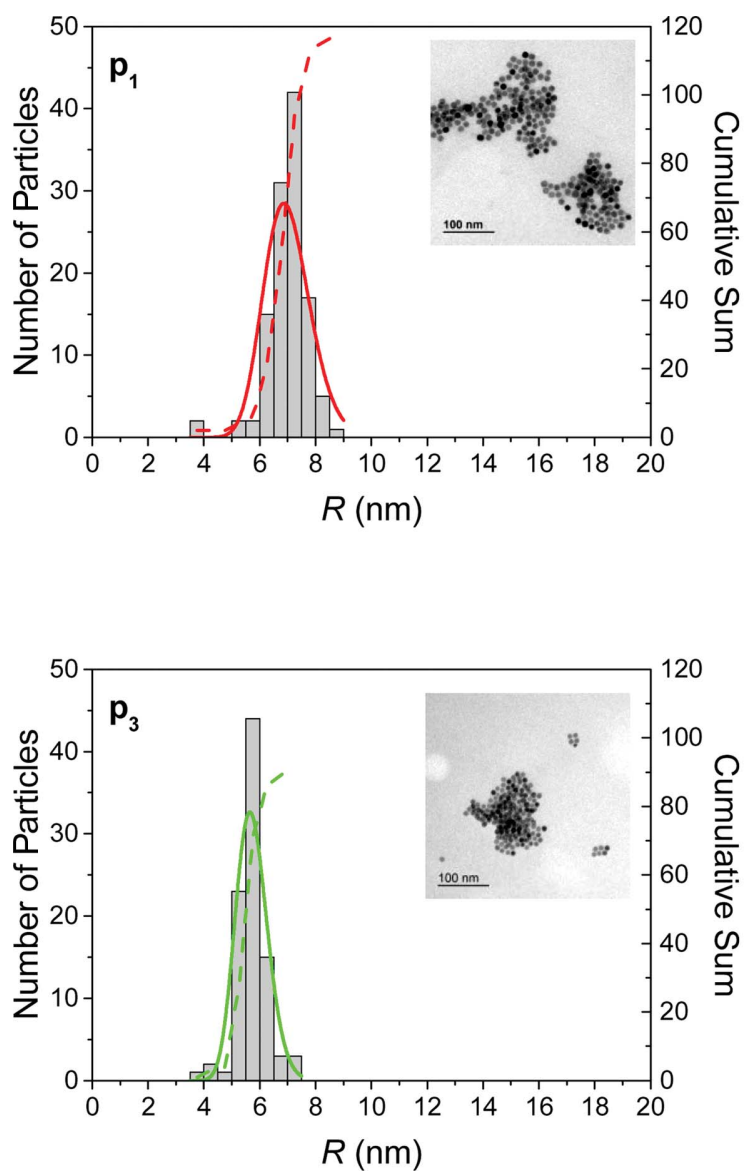

7. Type of iron oxide within the particles

All samples of the series have the same XANES spectrum at the $K$ edge of iron as can be seen in Fig. 7(a). The spectrum of magnetic nanoparticle cores exhibits all features of the XANES spectrum of maghemite (Schimanke \& Martin, 2000;
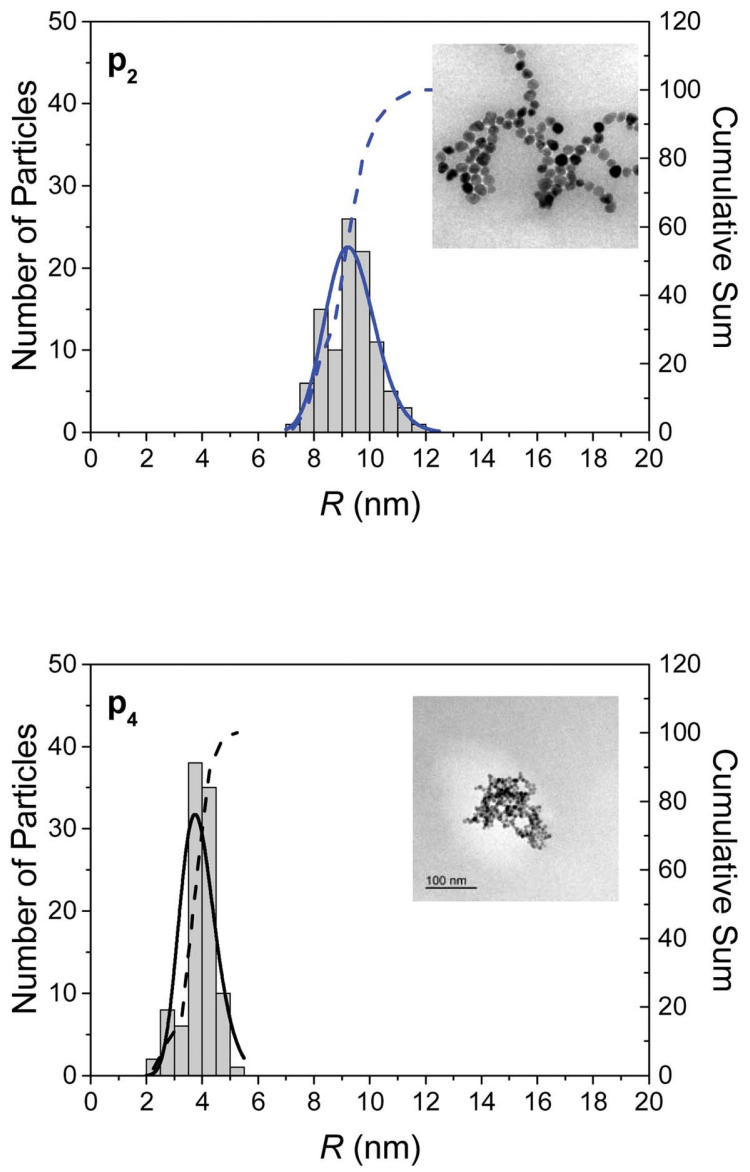

Figure 6

TEM results of particles $\mathrm{p}_{1}, \mathrm{p}_{2}, \mathrm{p}_{3}$ and $\mathrm{p}_{4}$. Histograms represent the number of particles with the radii derived from the pictures in the insets. Displayed are the partial differential functions (solid lines) from lognormal curve fits of the histograms and the cumulative sums from the histograms (dashed lines).

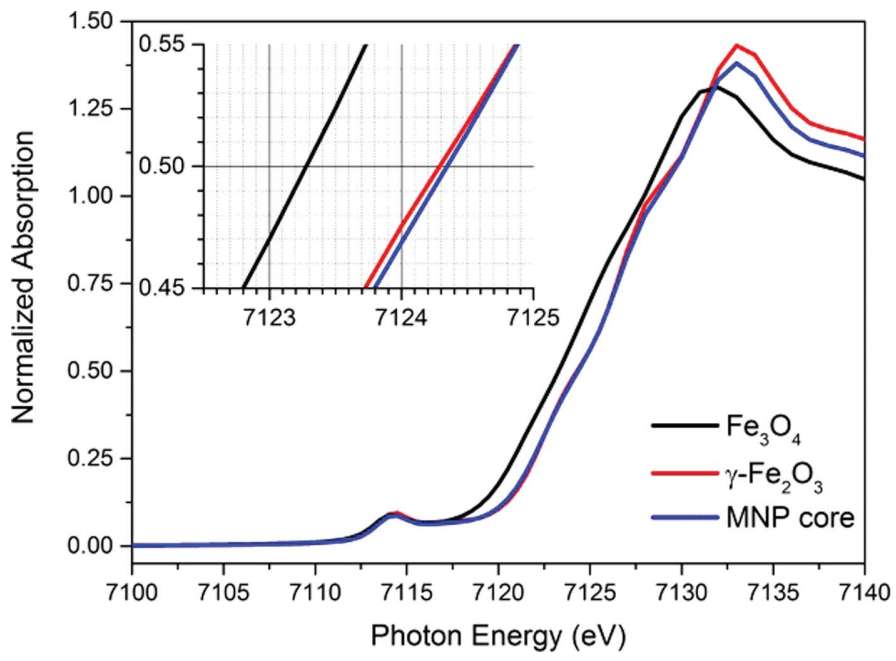

(a)

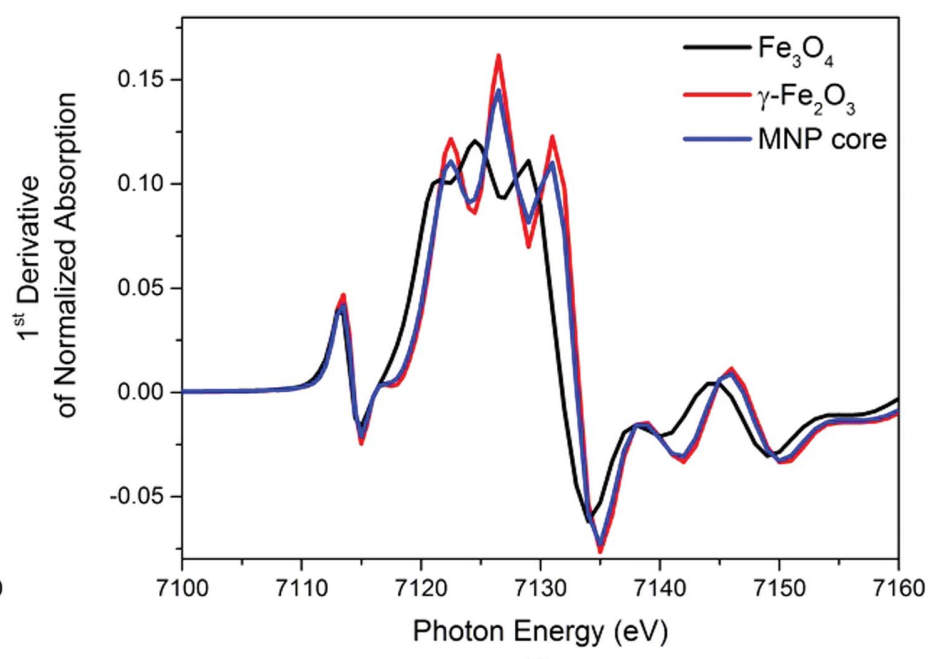

(b)

Figure 7

XANES of $\mathrm{p}_{3}$, which is representative for all particle cores of the series. The spectral shapes and the peak positions identify the cores unambiguously as $\gamma-\mathrm{Fe}_{2} \mathrm{O}_{3}$, both in the edge-step normalized spectrum $(a)$ and in the first derivative of the absorption spectrum $(b)$. 
Carta et al., 2013). This is especially recognizable in the first derivative of the XANES spectrum in Fig. 7(b). The main peak and its three distinctive maxima have exactly the same positions as those of the $\gamma-\mathrm{Fe}_{2} \mathrm{O}_{3}$ reference. This means that no $\mathrm{Fe}^{\mathrm{II}}$ is present in the sample in detectable amounts. Moreover, the shape of the MNP core spectra follows almost exactly the shape of the maghemite reference spectrum right above the absorption edge. This part of the XANES contains structural information according to the multiple-scattering theory (Rehr \& Albers, 2000). Thus, the iron oxide cores consist only of maghemite phase, which is an inverse spinel structure with $\mathrm{Fe}^{\mathrm{III}}$ ions only and vacancies in the cation sublattice for charge neutralization. The findings on the internal structure and composition of the particles' cores are in agreement with the aims of the synthesis route chosen.

\section{Conclusions}

The X-ray techniques applied here delivered valuable results for the MNP systems under investigation. SAXS gave a thorough characterization of single-core and multi-core MNPs in terms of core size and size distribution, while XANES proved that the desired crystalline structure and oxidation state of the iron ions was indeed achieved. Information on the inner structure of the MNP, as well as on the morphology of the system, i.e. core size, size distribution of core aggregates and number of cores within an aggregate, is of extreme interest for use in subsequent calculations and modelling of magnetic properties of these systems. The single-core spherical particles are a starting point for a reference material aiming for the minimization of the effects connected to the shape anisotropy and intra-particle dipolar interactions. The desired narrow size distribution and the lack of significant numbers of aggregated cores can be easily verified by SAXS, as we have shown. A further motivation for this study was to identify spherical multi-core MNPs with the potential to act as a magnetic reference material, with minimal shape anisoptropy and dipolar interaction properties that can be controlled via the size of the agglomerates and the number of cores inside such an object. SAXS in combination with SLS delivered estimates of these morphological parameters. This information can be translated into, for example, mean core distances inside the multi-core. This is of great importance for the modelling of such magnetically interacting cores, which often give rise to bulk magnetization behaviour that is hard to understand without such input. This kind of problem would strongly benefit from the use of a USAXS instrument, closing the information gap between SAXS and SLS data.

\section{Acknowledgements}

WS acknowledges funding from the European Union Seventh Framework Programme (FP7/2007-2013) under grant agreement No. 604448.

\section{References}

Bogren, S., Fornara, A., Ludwig, F., Morales, M. D., Steinhoff, U., Hansen, M. F., Kazakova, O. \& Johansson, C. (2105). Int. J. Mol. Sci. 16, 20308-20325.

Bohara, R. A., Thorat, N. D. \& Pawar, S. H. (2016). RSC Adv. 6, 43989-44012.

Bonini, M., Fratini, E. \& Baglioni, P. (2007). Mater. Sci. Eng. C, 27, 1377-1381.

Breßler, I., Kohlbrecher, J. \& Thünemann, A. F. (2015). J. Appl. Cryst. 48, 1587-1598.

Carta, D., Corrias, A., Falqui, A., Brescia, R., Fantechi, E., Pineider, F. \& Sangregorio, C. (2013). J. Phys. Chem. C, 117, 9496-9506.

Ferretti, R., Zhang, J. W. \& Buffle, J. (1998). J. Colloid Interface Sci. 208, 509-517.

Görner, W., Hentschel, M. P., Müller, B. R., Riesemeier, H., Krumrey, M., Ulm, G., Diete, W., Klein, U. \& Frahm, R. (2001). Nucl. Instrum. Methods Phys. Res. Sect. A, 467-468, 703-706.

Guinier, A. \& G. Fournet, G. (1955). Small-Angle Scattering of $X$-rays. New York: John Wiley and Sons.

Hamilton, W. C. (1965). Acta Cryst. 18, 502-510.

Karimi, Z., Karimi, L. \& Shokrollahi, H. (2013). Mater. Sci. Eng. C, 33, 2465-2475.

Lartigue, L., Hugounenq, P., Alloyeau, D., Clarke, S. P., Lévy, M., Bacri, J. C., Bazzi, R., Brougham, D. F., Wilhelm, C. \& Gazeau, F. (2012). ACS Nano, 6, 10935-10949.

Lin, M. Y., Lindsay, H. M., Weitz, D. A., Ball, R. C., Klein, R. \& Meakin, P. (1989). Nature, 339, 360-362.

Lu, A. H., Salabas, E. L. \& Schüth, F. (2007). Angew. Chem. Int. Ed. 46, 1222-1244.

Odio, O. F., Lartundo-Rojas, L., Santiago-Jacinto, P., Martínez, R. \& Reguera, E. (2014). J. Phys. Chem. C, 118, 2776-2791.

O’Grady, K. \& Bradbury, A. (1983). J. Magn. Magn. Mater. 39, 91-94.

Orthaber, D., Bergmann, A. \& Glatter, O. (2000). J. Appl. Cryst. 33, 218-225.

Rehr, J. J. \& Albers, R. C. (2000). Rev. Mod. Phys. 72, 621-654.

Riesemeier, H., Ecker, K., Görner, W., Müller, B. R., Radtke, M. \& Krumrey, M. (2005). X-ray Spectrom. 34, 160-163.

Roca, A. G., Veintemillas-Verdaguer, S., Port, M., Robic, C., Serna, C. J. \& Morales, M. P. (2009). J. Phys. Chem. B, 113, 7033-7039.

Salas, G., Casado, C., Teran, F. J., Miranda, R., Serna, C. J. \& Morales, M. P. (2012). J. Mater. Chem. 22, 21065-21075.

Schimanke, G. \& Martin, M. (2000). Solid State Ionics, 136-137, 12351240.

Teixeira, J. (1988). J. Appl. Cryst. 21, 781-785.

Yoon, S. (2015). Hyperfine Interact. 231, 21-28. 